\title{
Feasibility of transnasal endoscopy in screening for esophageal and gastric varices in patients with chronic liver disease
}

\section{(ㄷ)(1) $\odot$}

\author{
Authors \\ Abreu Ferrari, Vitor Nunes Arantes \\ Institution \\ Hospital das Clínicas, Federal University of Mimas Gerais, \\ Alfa Institute of Gastroenterology, Belo Horizonte, Minas \\ Gerais, Brazil
}

Anderson Antônio de Faria, Carlos Alberto Freitas Dias, Luciana Dias Moetzsohn, Silas de Castro Carvalho, Tereza

submitted 23.10.2016

accepted after revision 24.3.2017

\author{
Bibliography \\ DOI https://doi.org/10.1055/s-0043-107781 | \\ Endoscopy International Open 2017; 05: E646-E651 \\ (c) Georg Thieme Verlag KG Stuttgart · New York \\ ISSN 2364-3722
}

Corresponding author

Anderson Antônio de Faria, Universidad Federal de Minas

Gerais - Medicine, Avenida Professor Alfredo Balena, 100.

Santa Efigenia Instituto Alfa de Gatroenterologia, Belo

Horizonte Belo Horizonte Minas Gerais, 31270-901 Brazil

Fax: +3409-9408

andersonpita@yahoo.com.br

\section{ABSTRACT}

Background and study aims Screening for esophageal and gastric varices is indicated for patients with portal hypertension or cirrhosis. Typically, conventional endoscopy is used; however, the need for sedation increases the costs and risks, especially in cirrhotic patients. Use of transnasal endoscopy with an ultrathin endoscope enables study of the upper gastrointestinal tract without the need for sedation. The objective of this study is to evaluate the feasibility of transnasal endoscopy in screening for esophageal and gastric varices in patients with chronic liver disease.

Patients and methods This was a prospective study in which transnasal endoscopy was carried out in patients with cirrhosis or portal hypertension who had indications for screening of esophageal and gastric varices. The following variables were evaluated: demographical data, duration of procedure, patient tolerance and acceptance, adverse events (AEs), endoscopic findings and interobserver agreement related to portal hypertension alterations (kappa index).

Results A total of 50 patients entered the study. The most common cause of liver disease was chronic viral hepatitis (66\%). Among the cirrhotic patients, most of the patients were Child-Pugh A (74\%). In 5 patients (10\%), nasal intubation was not possible. Two patients (4\%) experienced minor epistaxis. Tolerance was excellent or good in $92 \%$ according with a visual analogic scale. In 16 patients (32\%), esophageal varices were detected and in 2 patients (4\%) gastric varices were detected. The mean duration of the procedure was 7 minutes.

Conclusions Transnasal endoscopy is feasible, effective and well tolerated for screening of esophageal and gastric varices in patients with chronic liver disease. It can be performed in outpatient clinics safely and without the use of sedation.

\section{Introduction}

Esophageal and gastric varices are present in approximately half of patients with cirrhosis [1]. The increased portal resistance observed in cirrhotic patients is the initial event that leads to collateral formation in the portal system and diverts blood flow from the liver to the systemic circulation [2]. The normal hepatic venous pressure gradient (HVPG) varies between 3 and $5 \mathrm{mmHg}$. Portal hypertension occurs when this gradient is greater than $5 \mathrm{mmHg}$ [3].
Portal hypertension and varices formation can also occur in the absence of cirrhosis. Some diseases, such as hepatosplenic schistosomiasis, affect vascular resistance in the liver or the patency of splanchnic vessels, leading to deviation of the flow and formation of esophageal and gastric varices [4]. Non-cirrhotic patients with portal hypertension are also at risk of hemorrhagic complications, therefore primary and secondary prevention measures are also indicated for this population [5].

Rupture of varices is the major cause of death in cirrhotic patients, responsible for high rates of morbidity and mortality [6]. The average risk of variceal rupture is approximately $12 \%$ [7]. 
Despite advances in drug and endoscopic therapy, variceal rupture still has a mortality rate between $20 \%$ and $30 \%[7,8]$.

Currently screening for esophageal and gastric varices with the aim of instituting measures to prevent upper gastrointestinal hemorrhage is recommended in patients with advanced chronic liver disease. The latest Baveno consensus (Baveno VI) recommends esophagogastroduodenoscopy (EGD) for patients with chronic liver disease to screen for varices. An exception is made for those with an elastogram less than $20 \mathrm{kPa}$ and a platelet count of more than 150,000 , who in theory do not require screening [9].

EGD is the best diagnostic method for screening of esophageal and gastric varices [9]. However, the need for sedation increases costs, affects labor productivity and introduces a small but not insignificant risk of AEs, particularly in patients with decompensated cirrhosis [10].

In recent years, interest in transnasal endoscopy (TNE) has increased [11-13]. However, few studies have evaluated its use in assessment of portal hypertension complications. The objective of this study was to evaluate the feasibility of TNE in screening of esophageal and gastric varices in patients with chronic hepatic disease.

\section{Patients and methods}

This is a prospective case series performed from March 2014 to January 2016 in Belo Horizonte, Brazil, that enrolled patients with chronic hepatic disease referred from the Viral Hepatitis or General Hepatology outpatient clinic who had an indication for screening for esophageal and gastric varices with EGD.

Inclusion criteria were: age between 18 and 80 years; clinical, laboratory, radiological, or histological diagnosis of liver cirrhosis or portal hypertension; referral to endoscopy unit for screening or monitoring of portal hypertension complications, according to Baveno V (before September 2015) or Baveno VI (after September 2015) recommendations; and agreement to participate in the study with a signed consent form. The study was approved by the Institutional Review Board at the Federal University of Minas Gerais and was registered in clinicaltrials. gov: NCT02767206. Patients who had any of the following criteria were excluded: previous nasal surgery; coagulation disorders with an: international normalized ratio (INR) higher than 2 or platelet count less than 40,000; use of oral anticoagulants, heparin, or antiplatelet agents; history of repeated epistaxis; rhinitis; nasal or oropharynx anatomical abnormality, current upper airway infection.

The examinations were carried out in the endoscopy unit at the Alfa Institute of Gastroenterology of the Federal University of Minas Gerais and in the Jenny de Andrade Faria Institute, an outpatient clinic attached to the hospital. Three endoscopists with experience in TNE performed the procedure. Images of the esophagus (distal third), stomach (fundus, body, and antrum), and duodenum (first and second portion) were recorded. Subsequently, 2 independent endoscopists reviewed the images at different times, to calculate the inter-observer agreement.

\section{TNE procedure}

An ultrathin endoscope (Fujifilm EG350N Fujifilm Co, Japan), with a diameter of $5.9 \mathrm{~mm}$ was used, coupled to a Fujifilm 4450 image processor.

The examination was performed in the left lateral decubitus position. Patients were monitored for heart rate and pulse oximetry. They received 2 puffs of lidocaine (10\%) in the orophar$\mathrm{ynx}$ and 5 drops of a nasal vasoconstrictor (naphazoline hydrochloride $0.5 \mathrm{mg} / \mathrm{mL}$ ). About 5 minutes later, lidocaine gel (2\%) was applied in the nostril chosen for introduction of the endoscope. Sedative agents were not employed. The decision as to which nostril was used depended on the local examination, taking into account factors such as patency and diameter. If intubation of the nostril was not achieved, intubation through the other nostril was attempted. If both were unsuccessful, an oral route was chosen, also without sedation.

The hypopharynx, esophagus, stomach, and duodenum were screened and the examination was considered complete when the second duodenal portion was reached. Esophageal and gastric varices were searched during the introduction and removal of the endoscope, but classification occurred during the withdrawal of the endoscope. Esophageal varices found were classified as follows: small caliber (up to $5 \mathrm{~mm}$ with minimum elevation in the esophageal mucosa), medium caliber (tortuous veins occupying less than $33 \%$ of the esophageal lumen), and large caliber (occupying more than $33 \%$ of the esophageal lumen) [14]. The gastric varices were classified as GOV1, GOV2, IGV1, and IGV2, according to Sarin Classification [15].

\section{Variables analyzed}

The following data were analyzed: age; gender; duration of the examination; etiology of liver disease; Child-Pugh classification; patient's preference - TNE or per-oral endoscopy (for those who had already undergone a conventional endoscopy); complications rate; endoscopic findings particularly for alterations related to portal hypertension (esophageal varices, red spots, gastric varices, hypertensive gastropathy.

The endoscopists rated patient tolerance to the exam as "excellent," "good," "fair," or "poor." Tolerance was also rated by a patient, using an internationally validated visual analog scale (VAS). This scale classifies numerically the intensity of pain or discomfort using a line numbered from $0-10$. At one end of the line ("0") "no pain or discomfort" is pointed out and, on the other end ("10"), is the "worst discomfort or pain" [11]. After the examination, an independent investigator asked each patient to indicate, using the VAS scale, the level of pain or discomfort experienced during the procedure. The answers were separated for analysis in the following sequence: 0 to 2 , minimal or no discomfort; 3 to 5 , medium discomfort; 6 to 8 , moderate discomfort; and 9 or 10 , intense pain or discomfort.

The kappa index was used to calculate the interobserver agreement related to the endoscopic findings. If the kappa was higher than 0.00 and lower than 0.40 the concordance was poor; fair if higher than 0.40 and lower than 0.75 ; very good if higher than 0.75 and less than 1.00; perfect if equal to $1.00[16]$. 
- Table 1 Baseline characteristics of 50 patients who underwent transnasal endoscopy.

\begin{tabular}{|l|c|}
\hline Variables & $\mathbf{n = 5 0}$ \\
\hline Age (average \pm standard deviation) & $54 \pm 12$ \\
\hline Gender & $27(54 \%)$ \\
\hline - Male & $23(46 \%)$ \\
\hline - Female & \\
\hline Etiology of liver disease & $22(44 \%)$ \\
\hline - Hepatitis C & $11(22 \%)$ \\
\hline - Hepatitis B & $6(12 \%)$ \\
\hline - Alcohol & $5(10 \%)$ \\
\hline - Cryptogenic & $4(8 \%)$ \\
\hline - Schistosomiasis & $2(4 \%)$ \\
\hline - Primary sclerosing cholangitis & $n=35$ \\
\hline Staging of cirrhotic patients & $26(74 \%)$ \\
\hline - Child-Pugh A & $7(20 \%)$ \\
\hline - Child-Pugh B & $2(6 \%)$ \\
\hline - Child-Pugh C & $153 \pm 69$ \\
\hline Platelets (average \pm standard deviation) $\times 100.000$ & $1.10( \pm 0.19)$ \\
\hline - INR ${ }^{1}$ (average \pm standard deviation) & \\
\hline 1 international normalized ratio & \\
\hline
\end{tabular}

\section{Results}

\section{Baseline characteristics of patients}

- Table 1 shows the general characteristics of patients enrolled in the study. TNE was carried out in 50 patients and 27 (54\%) were men. The age had a normal distribution, with a mean of 54 years (standard deviation \pm 12 ), ranging from 27 to 79 years, with $75 \%$ of the patients aged less than 65 years.

The most common etiology of liver disease was hepatitis $C$ (22 patients, $44 \%$ ), followed by hepatitis B (11 patients, $22 \%$ ), and by alcohol ( 6 patients, $12 \%)$. Among the 50 patients, 35 (70\%) had cirrhosis and 15 (30\%) had chronic liver disease without cirrhosis. The cirrhotic patients were staged according to the Child-Pugh classification, the majority being Child-Pugh A (74\%) ( Table 1$)$.

In 45 of 50 patients (90\%), the nostril was intubated with success. In 5 patients nasal intubation failed for the following reasons: pain or discomfort (2 patients), narrow size or anatomical variations (3 patients). In these patients, examination was carried out by the oral route, without sedation.

Thirty patients $(60 \%)$ had already undergone conventional EGD in the past. They were asked about the preferred route: 21 patients (70\%) indicated TNE as the preferred approach.

- Fig. 1 shows the evaluation of TNE pain/discomfort intensity according to the examiner or the patient. Tolerance of the

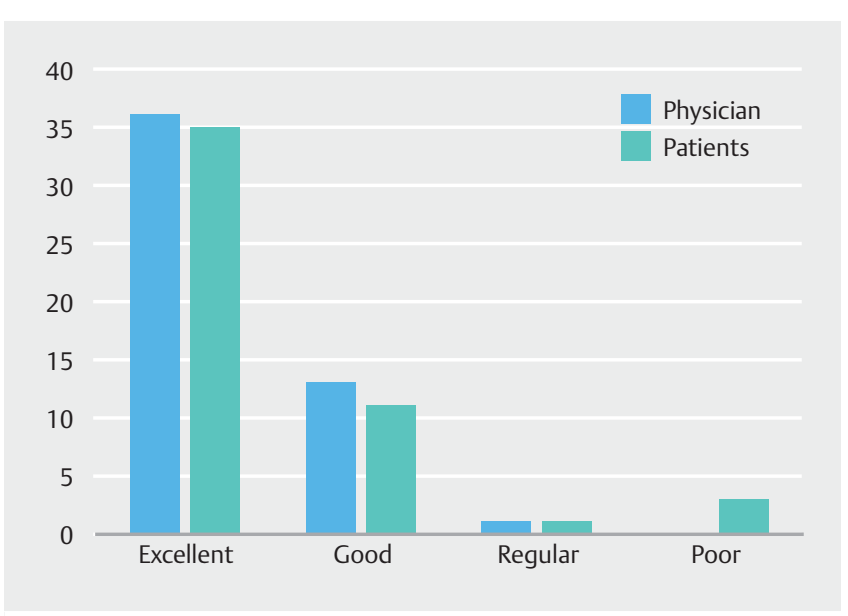

- Fig. 1 Physician and patient evaluation of tolerability of transnasal endoscopy.

examination, assessed by the examiner, was considered "excellent" in 36 patients (72\%), "good" in 13 patients (26\%), and "fair" in 1 patient ( $2 \%)$.

Patients assessed their tolerance of the examination using the VAS. According to this scale, $70 \%$ of the patients reported no discomfort (VAS from 0 to 2); 11 patients (22\%), slight discomfort (VAS from 3 to 5); 1 patient (2\%), moderate discomfort (VAS 6 to 8 ); and 3 patients (6\%) reported poor tolerance (VAS 9 to 10 ) (> Fig. 1 ).

The duration of the procedure had an uneven distribution, with a median of 7 minutes ( $95 \% \mathrm{Cl}: 7$ min $13 \mathrm{~s}$ to 8 min $9 \mathrm{~s}$ ). In $75 \%$ of patients, the examination was completed within 10 minutes. In all cases, a complete endoscopy up to the duodenum (second portion) was successfully carried out.

\section{Endoscopy findings}

Among 15 patients with portal hypertension without cirrhosis, only 1 had esophageal varices (6.6\%), while 16 cirrhotic patients out of 35 had esophageal varices (45\%). Esophageal varices were graded as small-caliber in 14 patients (40\%), medium size in $3(8.5 \%)$, and large-caliber in 1 (2.8\%). Four patients (11.4\%) presented with mild portal hypertensive gastropathy (PHG) and 1 individual had moderate PHG (2.8\%). Red spots were found in 3 patients ( $8.5 \%$ ) and gastric varices in 2 individuals $(5.7 \%)$ ( $>$ Table 2$)$.

Two patients (4\%) experienced self-limited epistaxis. In both cases, epistaxis were minor and self-limited and did not affect patient outcome. One of these patients was Child-Pugh C, had 88,000 platelets and prothrombin activity of $38 \%$ (INR: 1.88). The other patient was Child-Pugh A, with 113,000 platelets and prothrombin activity of $68 \%$ (INR: 1.18 ). No other complications were noted.

Recorded endoscopic images with portal hypertension were evaluated by 2 independent endoscopists, for calculation of interobserver agreement. For detection and classification of esophageal varices, the kappa value for agreement was 0.72 (intermediate); for gastric varices, the correlation was perfect (kappa $=1$ ). Red spots and PHG were respectively kappa 1 (per- 
- Table 2 Distribution of endoscopic findings related to portal hypertension of 35 patients with cirrhosis.

\begin{tabular}{|l|l|l|l|l|l|l|l|l|}
\hline \multicolumn{2}{|c|}{ Child-Pugh } & \multicolumn{3}{|c|}{ Esophageal varices } & \multicolumn{3}{|c|}{ Hypertensive gastropathy } & Red spots \\
\hline & Small & Medium & Large & Mild & Moderated & Severe & \\
\hline A & 9 & - & - & - & - & - & - \\
\hline B & 4 & 1 & - & 3 & - & - & 2 & - \\
\hline C & 1 & 2 & 1 & 1 & 1 & - & 1 & 1 \\
\hline Overall & 14 & 3 & 1 & 4 & 1 & 0 & 3 \\
\hline
\end{tabular}

fect) and 0.8 (very good). Images of the main endoscopic findings were recorded ( $\triangleright$ Fig. 2 ).

\section{Discussion}

This study aimed to prospectively evaluate the feasibility of TNE for screening of esophageal and gastric varices in patients with chronic liver disease. TNE proved to be a safe and well-tolerated method, and allowed for reliable diagnosis of major changes related to portal hypertension. All procedures were performed without sedation, enabling a shorter recovery time and preventing AEs from sedative drugs.

The main causes for liver disease in our population were hepatitis C, hepatitis B, and alcoholism. None of the patients had nonalcoholic steatohepatitis (NASH)-associated cirrhosis, which has recently become one of the main causes of cirrhosis worldwide [17].

The success rate of nasal intubation was high (90\%) and the rate of AEs was low (4\%), similar to previously reported data [18], showing that TNE is feasible and safe in patients with liver disease. Nasal pain or small size of the nostril prevented success with TNE in 5 patients (10\%), similar to other studies $[18,19]$. TNE was completed satisfactorily reaching the second portion of the duodenum in all cases, as demonstrated in previous studies [20].

In our study, $70 \%$ of patients who had previously undergone conventional EGD indicated a preference for TNE if a repeated endoscopic examination was required in the future. Stroppa et al. [21] conducted a comparative study in which patients were submitted to 2 exams on different days, TNE (without sedation) or EGD (under sedation). The authors reported that $87 \%$ of their patients also preferred TNE, similar to data from other studies [22,23].

Murata et al. [24] compared oral and transnasal EGD in 124 patients and observed that TNE reduced discomfort compared to oral access. In another study, 111 patients were divided into 2 groups to compare activation of the autonomic nervous system when access was transnasal (55 patients) or oral (56 patients). Lower levels of sympathetic nervous system stimulation were observed in the group of TNE patients, resulting in lower elevation in systolic pressure and heart rate [25]. Choe et al. screened 84 patients with portal hypertension [18] who underwent both TNE and oral EGD. The objective was to assess TNE accuracy, tolerance and AE. Accuracy in diagnosis of esophageal varices was $98 \%$. Tolerance to TNE was higher than tolerance to per-oral EGD $(P=0.001)$. A total of $87 \%$ of their patients who underwent both procedures stated they preferred the transnasal approach. In our study, $92 \%$ of patients reported excellent or good tolerance to TNE endoscopy and $70 \%$ of them would be willing to repeat TNE instead of oral EGD if needed in the future.

Mean duration of the exam in our series $(7 \mathrm{~min})$ was lower than reported in other studies. Previous studies indicated that TNE exam was longer than conventional EGD [26]. However, those were initial studies and lack of experience has been suggested as a possible cause for a long procedure [20]. Craig et al. [27] directly compared the 2 methods and showed that procedure duration was shorter with TNE (15 vs. $20 \mathrm{~min}$ ) and that recovery time (7 vs. $37 \mathrm{~min}$ ) was shorter and pharmaceutical costs remarkably lower than for conventional EGD. Post-sedation recovery time after EGD corresponds to approximately $70 \%$ of the time required for the entire procedure [28]. The substantial reduction in total procedure duration and costs, increasing turnover of patients in the endoscopy unit, is attractive for the health care system (either public or private).

The current study was not intended to compare the accuracies of TNE and conventional EGD. Such comparative studies have been previously published. Jobe et al. demonstrated the diagnostic capacity of TNE for assessment of Barrett's esophagus, observing a difference only in the biopsy sample size, however, without affecting adversely the accuracy of the histological assessment [12]. For diagnosis of hiatal hernia and reflux esophagitis, transnasal TNE had the same accuracy as conventional EGD [29]. Arantes et al. performed a prospective study with 106 patients, assessing the role of TNE for detection of esophageal cancer in a high-risk population. The method had excellent diagnostic performance and was well tolerated by patients [11]. Although suction through the smaller working channel of the ultra-thin endoscope is more time-consuming than with standard gastroscopy, air insufflation is not different from routine EGD, therefore, our impression is that staging of varices is not impaired during TNE, an assumption supported by 2 studies [33, 34]. Aedo et al. found an excellent correlation between conventional EGD and TNE in detecting esophageal varices (Kappa: 0.90) [29]. Saeian et al. carried out a comparative study in 15 patients with cirrhosis, subjecting them to TNE and posteriorly to peroral EGD under sedation (on the same day). Both methods detected esophageal and gastric varices equally. Moreover, the 2 methodologies completely agreed in relation to the varices classification [30]. 

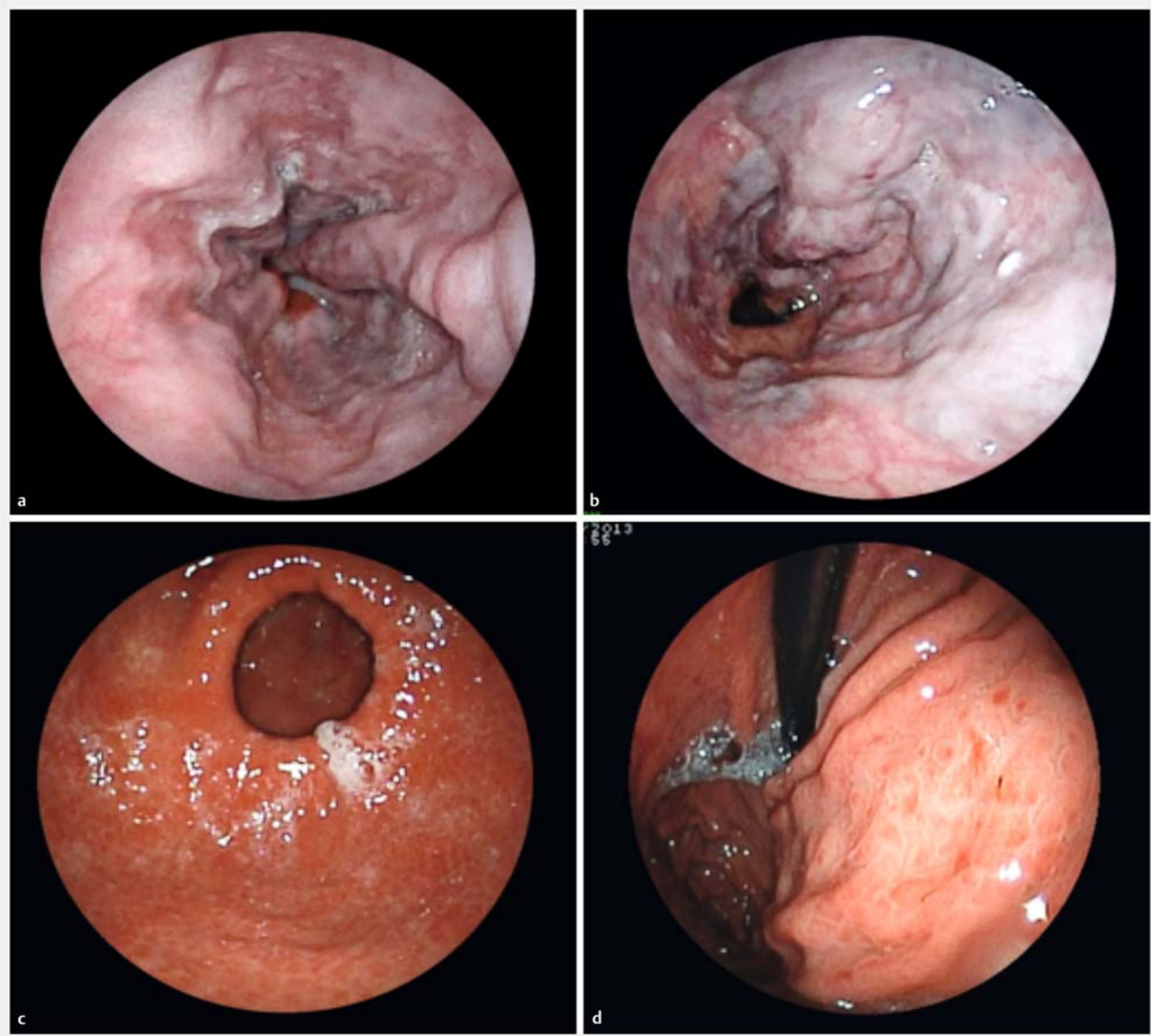

- Fig. 2 Endoscopic findings related to portal hypertension. a Esophageal varices of small and medium caliber. b Large-caliber esophageal varices with red spots. c, $\mathbf{d}$ Hypertensive gastropathy.

The only complication registered in this study (epistaxis, 4\%) was also noted in previous publications, with similar incidence rates: ranging from $0 \%$ to $5 \%[31,32]$. In all cases, epistaxis was mild and self-limited, with resolution within a few minutes after the procedure and without requiring any intervention.

Noninvasive methods for portal hypertension diagnosis have been proposed recently, such as esophageal capsule. A meta-analysis evaluated 16 studies and concluded that the current evidence does not allow an indication for use of the endoscopic capsule as a tool for screening varices in adults with cirrhosis, despite the low incidence of AEs [33]. Evaluation of the venous portal system with Doppler ultrasound also has been studied. Among the many indexes tested, the spleno-portal index was accurate in predicting presence or absence of esophageal varices in patients with compensated cirrhosis, but it does not al- low for accurate classification of the grade of varices, which is essential information when making a recommendation for treatment [34]. Use of serum markers of liver fibrosis were also analyzed, but were not successful in predicting the existence of varicose veins. In another study, liver stiffness (LS) and spleen size-to-platelet ratio risk score (LSPS) have been shown to predict esophageal varices in patients with chronic hepatitis $C$, but do not allow variceal staging, and endoscopic assessment is still required [35].

\section{Conclusion}

In conclusion, this study demonstrated that TNE using an ultrathin endoscope might be an alternative for variceal screening in patients with chronic liver disease. TNE was feasible, safe, effec- 
tive, and well tolerated in this population. In addition, it can be performed at outpatient clinics and without sedation. Additional studies directly comparing the costs involved will be important to encourage the incorporation of TNE in private and public health care systems.

\section{Competing interests}

None

\section{References}

[1] Boyer TD. Natural history of portal hypertension. Clin Liver Dis 1997; $1: 31-44, x$

[2] Bosch J, Pizcueta P, Feu F et al. Pathophysiology of portal hypertension. Gastroenterol Clin North Am 1992; 21: 1-14

[3] Garcia-Tsao G, Bosch J. Varices and Variceal Hemorrhage in Cirrhosis: A New View of an Old Problem. Clin Gastroenterol Hepatol 2015; 13 : $2109-2117$

[4] Okuda K. Non-cirrhotic portal hypertension versus idiopathic portal hypertension. J Gastroenterol Hepatol 2002; 17: (Suppl. 03): S204 S213

[5] Turon F, Casu S, Hernandez-Gea V et al. Variceal and other portal hypertension related bleeding. Best Pract Res Clin Gastroenterol 2013; 27: $649-664$

[6] Sarin SK, Lamba GS, Kumar M et al. Comparison of endoscopic ligation and propranolol for the primary prevention of variceal bleeding. $\mathrm{N}$ Engl J Med 1999; 340: 988 - 993

[7] D'Amico G, Pagliaro L, Bosch J. Pharmacological treatment of portal hypertension: an evidence-based approach. Semin Liver Dis 1999; 19: $475-505$

[8] Graham DY, Smith JL. The course of patients after variceal hemorrhage. Gastroenterology 1981; 80: 800-809

[9] de Franchis R. Expanding consensus in portal hypertension: Report of the Baveno VI Consensus Workshop: Stratifying risk and individualizing care for portal hypertension. J Hepatol 2015; 63: 743-752

[10] Triantafillidis JK, Merikas E, Nikolakis D et al. Sedation in gastrointestinal endoscopy: current issues. World J Gastroenterol 2013; 19: $463-481$

[11] Arantes V, Albuquerque W, Salles JM et al. Effectiveness of unsedated transnasal endoscopy with white-light, flexible spectral imaging color enhancement, and lugol staining for esophageal cancer screening in high-risk patients. J Clin Gastroenterol 2013; 47: 314-321

[12] Jobe BA, Hunter JG, Chang EY et al. Office-based unsedated small-caliber endoscopy is equivalent to conventional sedated endoscopy in screening and surveillance for Barrett's esophagus: a randomized and blinded comparison. Am J Gastroenterol 2006; 101: 2693 - 2703

[13] Tanuma T, Morita Y, Doyama H. Current status of transnasal endoscopy worldwide using ultrathin videoscope for upper gastrointestinal tract. Dig Endosc 2016; 28: (Suppl. 01): 25 - 31

[14] Averbach M. Endoscopia Digestiva Diagnóstico e Tratamento. Livro da Sobed. Revinter Rdj, editor 2013

[15] Sarin SK, Kumar A. Gastric varices: profile, classification, and management. Am J Gastroenterol 1989; 84: 1244 - 1249

[16] Sackett DLHR, Guyat GH, Tugwell P. Clinical epidemiology: a basic science for clinical medicine. ed. n, editor 1991

[17] Lee JY, Kim KM, Lee SG et al. Prevalence and risk factors of non-alcoholic fatty liver disease in potential living liver donors in Korea: a review of 589 consecutive liver biopsies in a single center. J Hepatol 2007; 47: $239-244$
[18] Choe WH, Kim JH, Ko SY et al. Comparison of transnasal small-caliber vs. peroral conventional esophagogastroduodenoscopy for evaluating varices in unsedated cirrhotic patients. Endoscopy 2011; 43: 649-656

[19] Atar M, Kadayifci A. Transnasal endoscopy: Technical considerations, advantages and limitations. World J Gastrointest Endosc 2014; 6: 41 48

[20] Trevisani L, Cifala V, Sartori S et al. Unsedated ultrathin upper endoscopy is better than conventional endoscopy in routine outpatient gastroenterology practice: a randomized trial. World J Gastroenterol 2007; 13: 906-911

[21] Stroppa I, Grasso E, Paoluzi OA et al. Unsedated transnasal versus transoral sedated upper gastrointestinal endoscopy: a one-series prospective study on safety and patient acceptability. Dig Liver Dis 2008; 40: $767-775$

[22] Murata A, Akahoshi K, Motomura Y et al. Prospective comparative study on the acceptability of unsedated transnasal endoscopy in younger versus older patients. J Clin Gastroenterol 2008; 42: 965 968

[23] Alexandridis E, Inglis S, McAvoy NC et al. Randomised clinical study: comparison of acceptability, patient tolerance, cardiac stress and endoscopic views in transnasal and transoral endoscopy under local anaesthetic. Aliment Pharmacol Ther 2014; 40: 467-476

[24] Murata A, Akahoshi K, Sumida Y et al. Prospective randomized trial of transnasal versus peroral endoscopy using an ultrathin videoendoscope in unsedated patients. J Gastroenterol Hepatol 2007; 22: 482 485

[25] Mori A, Ohashi N, Tatebe $\mathrm{H}$ et al. Autonomic nervous function in upper gastrointestinal endoscopy: a prospective randomized comparison between transnasal and oral procedures. J Gastroenterol 2008; 43: $38-44$

[26] Shaker R, Saeian K. Unsedated transnasal laryngo-esophagogastroduodenoscopy: an alternative to conventional endoscopy. Am J Med 2001; 111: Suppl 8A 153s-156s

[27] Craig A, Hanlon J, Dent J et al. A comparison of transnasal and transoral endoscopy with small-diameter endoscopes in unsedated patients. Gastrointest Endosc 1999; 49: 292-296

[28] Garcia RT, Cello JP, Nguyen MH et al. Unsedated ultrathin EGD is well accepted when compared with conventional sedated EGD: a multicenter randomized trial. Gastroenterology 2003; 125: 1606 - 1612

[29] Aedo MR, Zavala-Gonzalez MA, Meixueiro-Daza A et al. Accuracy of transnasal endoscopy with a disposable esophagoscope compared to conventional endoscopy. World J Gastrointest Endosc 2014; 6: 128 136

[30] Saeian K, Staff D, Knox J et al. Unsedated transnasal endoscopy: a new technique for accurately detecting and grading esophageal varices in cirrhotic patients. Am J Gastroenterol 2002; 97: 2246 - 2249

[31] Maffei M, Dumonceau JM. Transnasal esogastroduodenoscopy (EGD): comparison with conventional EGD and new applications. Swiss Med Wkly 2008; 138: $658-664$

[32] Tatsumi Y, Harada A, Matsumoto T et al. Current status and evaluation of transnasal esophagogastroduodenoscopy. Dig Endosc 2009; 21: $141-146$

[33] Colli A, Gana JC, Turner D et al. Capsule endoscopy for the diagnosis of oesophageal varices in people with chronic liver disease or portal vein thrombosis. Cochrane Database Syst Rev 2014: Cd008760

[34] Chakrabarti R, Sen D, Khanna V. Is non-invasive diagnosis of esophageal varices in patients with compensated hepatic cirrhosis possible by duplex Doppler ultrasonography? Indian J Gastroenterol 2016; 35: $60-66$

[35] Shibata S, Joshita S, Umemura T et al. Liver stiffness-spleen size-toplatelet ratio risk score detects esophageal varices in chronic liver disease. Springerplus 2016; 5: 998 